\title{
Epstein-Barr virus and carcinoma
}

SIR - A News and Views article by Rickinson ${ }^{1}$ raises very important questions on the biology of Epstein-Barr virus (EBV). Since our report ${ }^{2}$ that EBV genomes are regularly present in the epithelial cells of nasopharyngeal carcinoma (NPC), the discrepancy between in vitro experiments that failed to detect EBV receptors and the in vivo findings has been an important point of discussion. Rickinson discusses experiments by Sixby et al. ${ }^{3,4}$ on the detection of EBV genomes in exfoliated epithelial cells from throat washings and the finding that EBV can infect epithelial cells only if the virus is derived from throat washings, and not from tissue culture.

The data are interesting but not necessarily convincing. Sixby et al. have no evidence that the EBV-containing cells really originate from the throat, and their demonstration of the infection of epithelial cells involves an undefined system: throat washings contain a wide variety of components, including viruses of different kinds. Thus one must question their conclusion that mutants of EBV which are infectious to epithelial cells regularly exist or evolve. This would imply a very efficient and directed mutation of isolates of EBV obtained by collecting cell lines established from umbilical cord blood lymphocytes by immortalization with EBV from throat washings. The observation by Sixby may merely confirm the findings of Kehlifa and Menezes ${ }^{5}$, who were able to infect nonnatural target cells of EBV (EBV receptorfree cells) by preinfection or simultaneous infection with Sendai viruses. The samples used by Sixby et al. may have contained pseudotypes of EBV particles and other viruses infectious for epithelial cells ${ }^{6}$.

Our recent data, published too late for Rickinson to have been aware of, suggests an alternative interpretation of Sixby's data. We have found that EBV can replicate in the parotid gland of patients lacking antibodies to major EBV-related early antigens. This site of replication allows lifelong production of EBV without effective stimulation of the humoral antibody response because the EBV-producing cells are in the lumen of the salivary duct and not in contact with the bloodstream. Perhaps these cells are shed with the saliva and are then detected in the throat. The virus released may in fact be responsible for a constant supply by new infection of peripheral B lymphocytes with EBV genomes. Rickinson also mentions the production by Yiwan et al. of an EBVproducing cell line after fusion of Tupaia cells with lymphoblastoid cells ${ }^{8}$. This may be a model for the establishment of persistent low level lytic cycles of EBV in specific cells unable to suppress EBV replication in the way peripheral lymphocytes from convalescents seemingly do.

Besides lytic infection, EBV is characterized by its ability to undergo latency. It has long been known that in Burkitt's lymphoma and NPC biopsies, virus particles are only synthesized after one or two days of in vitro cultivation. As virus replication invariably leads to the lysis of the host cells, latency is a condition for a potential DNA tumour virus. In the case of NPC, the tumour cells are epithelial. The EBV genomes may enter these cells by mechanisms discussed above. However, mixed infections are very likely to kill the target cells. In addition, cells that can be infected by this mechanism should be located at the outer layers of the body and are therefore likely to be cells that have completed differentiation and are unable to live much longer. Aspirates of cells from the mucosa may contain such cells which may contain viral genomes but will even so undergo lysis ${ }^{9}$.

This does not mean that any transfer of EBV genomes to epithelial cells is an irreversible event leading invariably to the development of NPC. Nevertheless, the partial activation of EBV genes in lymphocytes harbouring otherwise latent EBV genomes and the consequent transfer of EBV genomes via EBV-mediated cell fusion to EBV receptor-negative epithelial cells ${ }^{10}$ would be a simple explanation for the presence of EBV genomes in submucosal epithelial cells. This mechanism would also offer a mechanistic explanation for the postulated activity of environmental agents as risk factors for the development of NPC.

\section{Max Von Petternkofer Institut}

HANS WOLF

der Ludwig-Maximilians-Universitat, Molekulare- und Tumorvirologie,

Petternkoferstr, $9 a$,

D-8000 München 2, FRG

1. Rickson, A. Nature 310, 99 (1984).

2. Wolf, H. et al. Nature new Biol. 244, 245 (1973)

3. Sixby et al. New Engl. J. Med. 310, 1225 (1984).

4. Sixby et al. Nature 306, 480 (1983).

5. Kehlifa \& Menezes in Nasopharyngeal Carcinoma Current Concepts (eds Prasad, U. et al.) 245 (1983).

6. Shapiro et al. Biochim. biophys. Actá 696, 19 (1981)

7. Wolf et al. J. Virol. 51, $795-798$ (1984).

8. Yiwan et al. Scient. sin. 27, 284 (1984).

9. Desgranges et al. Int. J. Cancer 29.87 (1982).

0. Bayliss \& Wolf Proc. natn. Acad. Sci. U.S.A. 78, 7162 (1981).

\section{Succession theory is too reductionist}

Sir - Bryan Finegan (Nature 312, $109-114 ; 1984)$ is right to point out that the pendulum of succession theory has swung too far from the holist view to the reductionist, and his article is welcome. Any attempt to categorize all pioneer species in terms of $r$-selection would be perverse, bearing in mind that the heterogeneous nature of the environments that pioneers colonize - from open water through various bare rock materials (sand, gravel, lava) to mature soils. The ability of textbooks to quote successively the 'standard succession', which always starts with lichens, and the $r$-selected char- acteristics of pioneers, has long puzzled me.

I would like to add two points. First, although he sensibly allows the mechanisms of facilitation, tolerance and inhibition to interact and occur throughout succession, there is a real sense in which facilitation is a first requirement.

Many, if not all, primary successions exhibit a qualitative shift at some stage from aquatic to terrestrial, nitrogen-free to nitrogen-sufficient, very low to adequate water-holding capacity. These shifts determine whether late-successional species can grow in the initial stages - oak trees do not grow in water - and are achieved typically by autogenic change. In other words, facilitation is a widespread event in the early stages of primary succession because: (1) Species vary in their ability to grow in typical primary succession environments; and (2) the act of growing in such environments induces modification (terrestrialization, $\mathbf{N}$ and organic matter inputs). After the shift has been achieved, however, other processes may be much more important.

Second, I think that it is important to point out that not all macronutrients are deficient in early primary succession. Because of the lack of mineral $\mathrm{N}$ source in most soils, $\mathrm{N}$ is almost always limiting but $P$ and $K$ are almost entirely soil-derived. $K$ is always entirely inorganic in soil, and may increase in availability due to inputs (particularly in maritime environments) and decomposition of minerals and decrease due to leaching and particulate loss. The overall trend is typically downwards. $\mathbf{P}$ is initially wholly inorganic, but over time declines in absolute amount, due to particulate loss and to a lesser extent leaching, while shifting gradually to organic forms. Most of the organic $P$ cycles very slowly, if at all, and the solution $P$ concentrations decline.

The net result of an increase in $\mathbf{N}$ availability and a decrease in $\mathbf{P}$ and $K$ supply should produce a brief period, some time after the initiation of a succession, when productivity is maximal. Interestingly, such a pattern is frequently observed, though not usually ascribed to such a cause.

Department of Biology, University of York, York YOI SDD, UK

\section{Disease and the elm decline}

SIR - I read the note by Peter Moore ${ }^{1}$ on the historical decline of elms with considerable interest. I pointed out elsewhere ${ }^{2}$ that the presence of elm bark beetle (Scolytus scolytus) remains in the fossil record does not prove that Dutch elm disease occurred, as the beetle may not always carry the fungal pathogen responsible for the disease. The only effective means of testing the disease hypothesis is to try to grow any 